\title{
Intensive care unit versus high-dependency care unit for patients with acute heart failure: a nationwide propensity score-matched cohort study
}

Hiroyuki Ohbe ${ }^{*} \mathbb{0}$, Hiroki Matsui and Hideo Yasunaga

\begin{abstract}
Background: A structure and staffing model similar to that in general intensive care unit (ICUs) is applied to cardiac intensive care unit (CICUs) for patients with acute heart failure. However, there is limited evidence on the structure and staffing model of CICUs. The present study aimed to assess whether critical care for patients with acute heart failure in the ICUs is associated with improved outcomes than care in the high-dependency care units (HDUs), the hospital units in which patient care levels and costs are between the levels found in the ICU and general ward.
\end{abstract}

Methods: This nationwide, propensity score-matched, retrospective cohort study was performed using a national administrative inpatient database in Japan. We identified all patients who were hospitalized for acute heart failure and admitted to the ICU or HDU on the day of hospital admission from April 2014 to March 2019. Propensity scorematching analysis was performed to compare the in-hospital mortality between acute heart failure patients treated in the ICU and HDU on the day of hospital admission.

Results: Of 202,866 eligible patients, 78,646 (39\%) and 124,220 (61\%) were admitted to the ICU and HDU, respectively, on the day of admission. After propensity score matching, there was no statistically significant difference in in-hospital mortality between patients who were admitted to the ICU and HDU on the day of admission (10.7\% vs. $11.4 \%$; difference, $-0.6 \% ; 95 \%$ confidence interval, $-1.5 \%$ to $0.2 \%)$. In the subgroup analyses, there was a statistically significant difference in in-hospital mortality between the ICU and HDU groups among patients receiving noninvasive ventilation ( $9.4 \%$ vs. $10.5 \%$; difference, $-1.0 \%$; $95 \%$ confidence interval, $-1.9 \%$ to $-0.1 \%$ ) and patients receiving intubation (32.5\% vs. $40.6 \%$; difference, $-8.0 \%$; $95 \%$ confidence interval, $-14.5 \%$ to $-1.5 \%$ ). There were no statistically significant differences in other subgroup analyses.

Conclusions: Critical care in ICUs was not associated with lower in-hospital mortality than critical care in HDUs among patients with acute heart failure. However, critical care in ICUs was associated with lower in-hospital mortality than critical care in HDUs among patients receiving noninvasive ventilation and intubation.

Keywords: Cardiac intensive care, Heart failure, Intensive care unit, High-dependency care unit, Propensity score, Administrative database

*Correspondence: hohbey@gmail.com

Department of Clinical Epidemiology and Health Economics, School of Public Health, The University of Tokyo, 7-3-1 Hongo, Bunkyo-ku,

Tokyo 1130033, Japan

\section{Background}

The numbers of critically ill patients with acute heart failure in cardiac intensive care units (CICU) have been dramatically increasing during the past few decades [1-3]. original author(s) and the source, provide a link to the Creative Commons licence, and indicate if changes were made. The images or other third party material in this article are included in the article's Creative Commons licence, unless indicated otherwise in a credit line to the material. If material is not included in the article's Creative Commons licence and your intended use is not permitted by statutory regulation or exceeds the permitted use, you will need to obtain permission directly from the copyright holder. To view a copy of this licence, visit http://creativecommons.org/licenses/by/4.0/. The Creative Commons Public Domain Dedication waiver (http://creativecommons.org/publicdomain/zero/1.0/) applies to the data made available in this article, unless otherwise stated in a credit line to the data. 
Management of acute heart failure using advanced drugs (e.g., intravenous vasodilators, inotropes, and vasopressors) and invasive organ supportive therapies (mechanical ventilation, mechanical circulatory support, and renal replacement therapy) has become a major focus of modern CICUs [4].

Based on the evidence gathered from general intensive care units (ICUs) [5-7], several academic societies have recommended a structure and staffing model for CICUs similar to that of general ICUs; e.g., intensivist staffing, a closed ICU model, and a nurse-to-patient ratio of $1: 1$ to $1: 2[4,8-10]$. However, evidence for these recommendations is limited when focusing on the structure and staffing model of CICUs [11, 12]. No clinical trials and only one observational study has shown that care by cardiac intensivists, compared with care by senior residents in internal medicine, is associated with reduced mortality in CICUs [13]. The benefit of critical care may vary depending on the severity of critical illness [7, 14], but no study has examined effect modification in the subset of patients with acute heart failure admitted to the CICU.

ICUs are the hospital units that provide the most advanced critical care, whereas high-dependency care units (HDUs) are the hospital units in which patient care levels and costs are between the levels found in the ICU and general ward [7]. CICUs are the hospital units that provide critical care for patients with cardiovascular diseases using various structure and staffing models, and many Japanese hospitals currently use some beds in their ICUs and HDUs as CICU beds for patients with acute heart failure because of the lack of insurance reimbursement criteria that specify what qualifies as a $\mathrm{CICU}[8,9$, 15]. Therefore, the current situation in Japan can provide a unique opportunity to assess the structure and staffing model of CICUs. Using a national inpatient database in Japan, we assessed the survival benefit of patients with acute heart failure who were admitted to the ICU versus HDU.

\section{Methods}

\section{Data source}

This was a nationwide, propensity score-matched, retrospective cohort study using the Diagnosis Procedure Combination database, a national inpatient database in Japan. The Institutional Review Board of The University of Tokyo approved this study (approval number, 3501-3; December 25, 2017).

The database contains discharge abstracts and administrative claims data from voluntarily participating hospitals [16]. In 2017, the database contained data for about $75 \%$ of all ICU beds and 70\% of all HDU beds in Japan [15]. This database includes the following patient-level data for all hospitalizations: demographic characteristics; diagnoses recoded with International Classification of Diseases, Tenth Revision (ICD-10) codes; daily procedures recorded using Japanese medical procedure codes; daily drug administrations; and admission and discharge status. A previous validation study for this database showed high specificity and moderate sensitivity for recorded diagnoses and high specificity and sensitivity for recorded procedures [17].

We also used facility information and statistics data from the Survey of Medical Institutions 2017 [18]. We combined these data with the Japanese Diagnosis Procedure Combination inpatient database using a specific hospital identifier. The Survey of Medical Institutions included the type of ward (e.g., general, ICU, or HDU), number of hospital beds in each ward, and hospital type (i.e., tertiary emergency hospital or academic hospital).

\section{Study population}

We identified all patients who were hospitalized for acute heart failure (ICD-10 codes I099, I110, I130, I132, I255, I420, I425-I429, I43x, or I50x) and were admitted to the ICU or HDU from April 1, 2014, to March 31, 2019. The sensitivity and specificity of the diagnosis of acute heart failure in the database was $68.8 \%$ and $97.5 \%$, respectively [17]. We excluded patients (i) aged $<15$ years, (ii) who were not admitted to the ICU or HDU on the day of hospital admission, and (iii) who were admitted to hospitals that could not be combined with data from the Survey of Medical Institutions 2017. All patients were followed up until they died or were discharged from the hospital.

\section{Treatment groups}

Patients who were admitted to the ICU on the day of hospital admission were defined as the ICU group. Patients who were admitted to the HDU on the day of hospital admission were defined as the HDU group. We compared the patients in the ICU group with those in the HDU group. The definition of ICU in this study was a separate unit providing critical care services with at least one physician on site $24 \mathrm{~h}$ per day, at least two intensivists working full-time (required only for resource-rich ICUs), around-the-clock nursing, the equipment necessary to care for critically ill patients, and a nurse-to-patient ratio of 1:2. An HDU, also called an "intermediate care unit" or "step-down unit", is area where patient care levels and costs are between the levels found in the ICU and in the general ward $[7,19,20]$. The definition of HDU in this study was almost the same as that of ICU, but an HDU had a nurse-to-patient ratio of $1: 4$ or 1:5 and no requirement for intensivist staffing. We present the Japanese procedure codes used to define ICUs and HDUs in Additional file 1: Table S1. 


\section{Outcomes}

The primary outcome was in-hospital mortality. The secondary outcomes were the length of hospital stay; length of ICU/HDU stay; total hospitalization costs (with 1 United States dollar equivalent to 110 Japanese yen); and complications including pneumonia, stroke, endoscopic hemostasis for gastrointestinal bleeding, catheterrelated bloodstream infection, and Clostridioides difficile infection.

\section{Covariates}

The covariates were age, sex, smoking history, body mass index at admission, Japan Coma Scale score at admission [21], physical function measured by the Barthel index score at admission [22], cognitive function before admission, home medical care before admission, location before admission, ambulance use, admission on a weekend (i.e., on Saturday or Sunday), comorbidities, Charlson comorbidity index score, treatments on the day of admission, and hospital characteristics.

\section{Statistical analysis}

We performed a propensity score-matching analysis to compare the outcomes between the ICU and HDU groups [23]. A multivariable logistic regression model using all the covariates listed in Table 1 was employed to compute the propensity scores for patients who were admitted to the ICU on the day of hospital admission. One-to-one nearest-neighbor matching without replacement was then performed for the estimated propensity scores using a caliper width set at $20 \%$ of the standard deviation of the propensity scores [23]. To assess the performance of the matching, the covariates were compared using standardized differences, with absolute standardized differences of $\leq 10 \%$ considered to denote negligible imbalances between the two groups [24]. After the propensity score matching, the primary and secondary outcomes for the two groups were assessed through a generalized linear model accompanied by cluster-robust standard errors with hospitals as the clusters. Differences and their 95\% confidence intervals were calculated with generalized linear models using the identity link function, irrespective of outcome types.

\section{Subgroup analyses}

We were interested in identifying the subsets of patients who would benefit most from a higher level of critical care. Therefore, based on previous studies [7, 14], we tested the potential for effect modification of ICU admission on in-hospital mortality according to treatment (respiratory support, intravenous vasodilators, diuretics, inotropes, vasopressors, renal replacement therapy, and mechanical circulatory support) on the day of admission. We performed these subgroup analyses among the propensity score-matched cohort created in the main analysis.

\section{Sensitivity analyses}

We performed two sensitivity analyses. First, the decision about which unit to which the patient would be assigned was made by the individual physician, with no specific criteria for one or the other, leading to likely confounding by indication. Therefore, we performed sensitivity analyses to compare primary and secondary outcomes excluding patients admitted to hospitals with both ICU beds and HDU beds. In these sensitivity analyses, the attending physicians had no choice of whether to admit patients to an ICU bed or HDU bed.

Second, there are two types of ICUs in Japan: resourcerich ICUs, which have two or more intensivists working as full-time employees, $\geq 20 \mathrm{~m}^{2}$ per ICU bed, and a medical engineer in the hospital $24 \mathrm{~h}$ per day; and other standard ICUs. The structure and staffing model in resource-rich ICUs are different from those in standard ICUs, especially in terms of the intensivist staffing requirement. Therefore, we performed sensitivity analyses to compare primary outcome between patients in resource-rich ICUs versus HDUs, standard ICUs versus HDUs, and resource-rich ICUs versus standard ICUs. For each sensitivity analysis, we repeated the propensity score-matching using the same method as in the main analysis.

All analyses were performed using Stata/MP 16.0 software (StataCorp, College Station, TX, USA). Continuous variables are presented as mean and standard deviation, and categorical variables are presented as number and percentage. All reported $P$ values were two-sided, and a $P$ value of $<0.05$ was considered statistically significant. Because of the potential for type I error due to multiple comparisons, findings for subgroup analyses should be interpreted as exploratory.

\section{Results}

In total, 202,866 eligible patients from 737 hospitals with ICU and/or HDU beds were enrolled during the 5-year study period (Fig. 1). Of these patients, 78,646 (39\%) were admitted to the ICU and 124,220 (61\%) were admitted to the HDU on the day of admission. Of 78,646 patients who were admitted to the ICU on the day of admission, 10,194 (13\%) were then transferred to the HDU. Of 124,220 patients who were admitted to the HDU on the day of admission, 4,589 (4\%) were then transferred to the ICU.

Table 1 shows the baseline characteristics before and after propensity score matching. In the original cohort, 
Table 1 Baseline characteristics before and after propensity score matching

\begin{tabular}{|c|c|c|c|c|c|c|}
\hline \multirow[t]{2}{*}{ Characteristics } & \multicolumn{3}{|c|}{ Before propensity score matching } & \multicolumn{3}{|c|}{ After propensity score matching } \\
\hline & $\begin{array}{l}\text { ICU } \\
(n=78,646)\end{array}$ & $\begin{array}{l}\text { HDU } \\
(n=124,220)\end{array}$ & ASD & $\begin{array}{l}\text { ICU } \\
(n=62,352)\end{array}$ & $\begin{array}{l}\text { HDU } \\
(n=62,352)\end{array}$ & ASD \\
\hline \multicolumn{7}{|l|}{ Age category, years } \\
\hline $15-59$ & $8623(11)$ & $9551(8)$ & 11 & $6012(10)$ & $6233(10)$ & 1 \\
\hline $60-69$ & $12,549(16)$ & $15,166(12)$ & 11 & $9097(15)$ & $9182(15)$ & 0 \\
\hline $70-79$ & $21,590(27)$ & $29,166(23)$ & 9 & $16,293(26)$ & $16,534(27)$ & 1 \\
\hline $80-89$ & $27,818(35)$ & $49,134(40)$ & 9 & $23,419(38)$ & $23,159(37)$ & 1 \\
\hline$\geq 90$ & $8066(10)$ & $21,203(17)$ & 20 & $7531(12)$ & $7244(12)$ & 1 \\
\hline Male & $45,539(58)$ & $65,536(53)$ & 10 & $34,891(56)$ & $35,036(56)$ & 1 \\
\hline \multicolumn{7}{|l|}{ Smoking history } \\
\hline Nonsmoker & $39,997(51)$ & $69,204(56)$ & 10 & $32,837(53)$ & $32,462(52)$ & 1 \\
\hline Current/past smoker & $28,244(36)$ & $39,154(32)$ & 9 & $21,569(35)$ & $21,841(35)$ & 1 \\
\hline Unknown & $10,405(13)$ & $15,862(13)$ & 1 & $7946(13)$ & $8049(13)$ & 1 \\
\hline \multicolumn{7}{|l|}{ Body mass index at admission, $\mathrm{kg} / \mathrm{m}^{2}$} \\
\hline$<18.5$ & $11,398(14)$ & $20,731(17)$ & 6 & $9487(15)$ & $9462(15)$ & 0 \\
\hline $18.5-24.9$ & $42,295(54)$ & $66,216(53)$ & 1 & $33,419(54)$ & $33,515(54)$ & 0 \\
\hline $25.0-29.9$ & $13,836(18)$ & $20,142(16)$ & 4 & $10,615(17)$ & $10,595(17)$ & 0 \\
\hline$\geq 30.0$ & $4786(6)$ & $6627(5)$ & 3 & $3653(6)$ & $3666(6)$ & 0 \\
\hline Missing & $6331(8)$ & $10,504(8)$ & 2 & $5178(8)$ & $5114(8)$ & 0 \\
\hline \multicolumn{7}{|l|}{ Japan Coma Scale score at admission } \\
\hline Alert & $55,363(70)$ & $90,007(72)$ & 5 & $45,087(72)$ & $45,263(73)$ & 1 \\
\hline Confusion & $14,730(19)$ & $25,663(21)$ & 5 & $11,892(19)$ & $11,696(19)$ & 1 \\
\hline Somnolence & $3886(5)$ & $4368(4)$ & 7 & $2638(4)$ & $2645(4)$ & 0 \\
\hline Coma & $4667(6)$ & $4182(3)$ & 12 & $2735(4)$ & $2748(4)$ & 0 \\
\hline \multicolumn{7}{|l|}{ Physical function at admission } \\
\hline Total/severe dependence (BI 0-60) & $44,788(57)$ & $75,717(61)$ & 8 & $36,189(58)$ & $35,992(58)$ & 1 \\
\hline Slight/moderate dependence (BI 61-99) & $2886(4)$ & $7479(6)$ & 11 & $2652(4)$ & $2515(4)$ & 1 \\
\hline Independent (B| 100) & $14,395(18)$ & $18,982(15)$ & 8 & $10,864(17)$ & $11,160(18)$ & 1 \\
\hline Missing & $16,577(21)$ & $22,042(18)$ & 8 & $12,647(20)$ & $12,685(20)$ & 0 \\
\hline \multicolumn{7}{|l|}{ Cognitive function before admission } \\
\hline No dementia & $62,514(79)$ & $87,997(71)$ & 20 & $48,040(77)$ & $48,328(78)$ & 1 \\
\hline Mild dementia & $11,281(14)$ & $23,981(19)$ & 13 & $9907(16)$ & $9729(16)$ & 1 \\
\hline Moderate/severe dementia & $4,851(6)$ & $12,242(10)$ & 14 & $4405(7)$ & $4295(7)$ & 1 \\
\hline Home medical care before admission & $4628(6)$ & $9537(8)$ & 7 & $4030(6)$ & $4044(6)$ & 0 \\
\hline \multicolumn{7}{|l|}{ Location before hospitalization } \\
\hline Home & $69,582(88)$ & $108,333(87)$ & 4 & $55,212(89)$ & $55,371(89)$ & 1 \\
\hline Other hospitals & $5069(6)$ & $5481(4)$ & 9 & $3430(6)$ & $3418(5)$ & 0 \\
\hline Nursing home & $3995(5)$ & $10,406(8)$ & 13 & $3710(6)$ & $3563(6)$ & 1 \\
\hline Ambulance use & $57,243(73)$ & $76,205(61)$ & 25 & $43,223(69)$ & $43,657(70)$ & 2 \\
\hline Admission on a weekend & $19,860(25)$ & $28,864(23)$ & 5 & $15,380(25)$ & $15,337(25)$ & 0 \\
\hline \multicolumn{7}{|l|}{ Comorbidities } \\
\hline Ischemic heart disease & $23,479(30)$ & $32,383(26)$ & 8 & $17,885(29)$ & $18,039(29)$ & 1 \\
\hline Diabetes mellitus & $25,442(32)$ & $35,323(28)$ & 9 & $19,500(31)$ & $19,644(32)$ & 1 \\
\hline Hypertension & $42,660(54)$ & $64,774(52)$ & 4 & $33,757(54)$ & $33,743(54)$ & 0 \\
\hline Hyperlipidemia & $19,133(24)$ & $26,527(21)$ & 7 & $14,714(24)$ & $14,788(24)$ & 0 \\
\hline Atrial flutter/fibrillation & $18,157(23)$ & $35,068(28)$ & 12 & $15,533(25)$ & $15,433(25)$ & 0 \\
\hline Chronic kidney disease & $13,750(17)$ & $19,434(16)$ & 5 & $10,384(17)$ & $10,327(17)$ & 0 \\
\hline Cancer & $3429(4)$ & $5646(5)$ & 1 & $2796(4)$ & $2767(4)$ & 0 \\
\hline Charlson comorbidity index & $1.4 \pm 1.4$ & $1.3 \pm 1.4$ & 7 & $1.4 \pm 1.3$ & $1.4 \pm 1.4$ & 0 \\
\hline
\end{tabular}


Table 1 (continued)

\begin{tabular}{|c|c|c|c|c|c|c|}
\hline \multirow[t]{2}{*}{ Characteristics } & \multicolumn{3}{|c|}{ Before propensity score matching } & \multicolumn{3}{|c|}{ After propensity score matching } \\
\hline & $\begin{array}{l}\text { ICU } \\
(n=78,646)\end{array}$ & $\begin{array}{l}\text { HDU } \\
(n=124,220)\end{array}$ & ASD & $\begin{array}{l}\text { ICU } \\
(n=62,352)\end{array}$ & $\begin{array}{l}\text { HDU } \\
(n=62,352)\end{array}$ & ASD \\
\hline \multicolumn{7}{|l|}{ Treatments on day of admission } \\
\hline \multicolumn{7}{|l|}{ Respiratory support } \\
\hline No supplemental oxygen & $13,848(18)$ & $21,594(17)$ & 1 & $12,005(19)$ & $12,106(19)$ & 0 \\
\hline Supplemental oxygen & $24,938(32)$ & $71,027(57)$ & 53 & $24,121(39)$ & $23,535(38)$ & 2 \\
\hline Noninvasive ventilation & $31,769(40)$ & $28,080(23)$ & 39 & $23,015(37)$ & $23,351(37)$ & 1 \\
\hline Intubation & $8091(10)$ & $3519(3)$ & 31 & $3211(5)$ & $3360(5)$ & 1 \\
\hline \multicolumn{7}{|l|}{ Intravenous vasodilator } \\
\hline Carperitide & $28,527(36)$ & $41,402(33)$ & 6 & $22,282(36)$ & $22,343(36)$ & 0 \\
\hline Nitrate & $33,780(43)$ & $35,205(28)$ & 31 & $23,851(38)$ & $24,297(39)$ & 2 \\
\hline Nicorandil & $5742(7)$ & $4894(4)$ & 15 & $3533(6)$ & $3598(6)$ & 1 \\
\hline Calcium-channel blocker & $9730(12)$ & $11,468(9)$ & 10 & $6980(11)$ & $7018(11)$ & 0 \\
\hline \multicolumn{7}{|l|}{ Diuretic } \\
\hline Intravenous furosemide & $52,132(66)$ & $84,840(68)$ & 4 & $42,043(67)$ & $41,961(67)$ & 0 \\
\hline Tolvaptan & $5980(8)$ & $11,935(10)$ & 7 & $5152(8)$ & $4945(8)$ & 1 \\
\hline \multicolumn{7}{|l|}{ Inotrope } \\
\hline Milrinone & $1059(1)$ & $1206(1)$ & 4 & $687(1)$ & $687(1)$ & 0 \\
\hline Pimobendan & $1473(2)$ & $2332(2)$ & 0 & $1203(2)$ & $1216(2)$ & 0 \\
\hline Dobutamine & $11,582(15)$ & $12,769(10)$ & 14 & $7580(12)$ & $7706(12)$ & 1 \\
\hline \multicolumn{7}{|l|}{ Vasopressor } \\
\hline Dopamine & $5254(7)$ & $5419(4)$ & 10 & $3205(5)$ & $3198(5)$ & 0 \\
\hline Noradrenaline & $5871(7)$ & $2782(2)$ & 25 & $2360(4)$ & $2399(4)$ & 0 \\
\hline \multicolumn{7}{|l|}{ Mechanical circulatory support } \\
\hline Intra-aortic balloon pumping & $1818(2)$ & $538(0)$ & 16 & $502(1)$ & $519(1)$ & 0 \\
\hline Extracorporeal membrane oxygenation & $375(0)$ & $101(0)$ & 8 & $88(0)$ & $97(0)$ & 0 \\
\hline \multicolumn{7}{|l|}{ Other treatment } \\
\hline Coronary angiography & $4252(5)$ & $2503(2)$ & 18 & $2116(3)$ & $2115(3)$ & 0 \\
\hline Percutaneous coronary intervention & $1317(2)$ & $601(0)$ & 12 & $539(1)$ & $545(1)$ & 0 \\
\hline Digoxin & $3128(4)$ & $4979(4)$ & 0 & $2535(4)$ & $2596(4)$ & 1 \\
\hline Intravenous beta-blockers & $3257(4)$ & $4056(3)$ & 5 & $2336(4)$ & $2365(4)$ & 0 \\
\hline Amiodarone & $3640(5)$ & $3712(3)$ & 9 & $2369(4)$ & $2431(4)$ & 1 \\
\hline Cardiac pacing & $1019(1)$ & $814(1)$ & 7 & $599(1)$ & $603(1)$ & 0 \\
\hline Intermittent renal replacement therapy & $3423(4)$ & $3370(3)$ & 9 & $2384(4)$ & $2430(4)$ & 0 \\
\hline Continuous renal replacement therapy & $2385(3)$ & $684(1)$ & 19 & $672(1)$ & $663(1)$ & 0 \\
\hline Antibiotics & $18,597(24)$ & $24,182(19)$ & 10 & $13,037(21)$ & $13,067(21)$ & 0 \\
\hline Morphine & $2421(3)$ & $2015(2)$ & 10 & $1530(2)$ & $1597(3)$ & 1 \\
\hline Red blood cell transfusion & $3352(4)$ & $3660(3)$ & 7 & $2108(3)$ & $2090(3)$ & 0 \\
\hline \multicolumn{7}{|l|}{ Hospital characteristics } \\
\hline Tertiary emergency hospital & $41,117(52)$ & $83,724(67)$ & 31 & $35,228(56)$ & $34,513(55)$ & 2 \\
\hline Academic hospital & $13,678(17)$ & $11,775(9)$ & 23 & $8643(14)$ & $8985(14)$ & 2 \\
\hline \multicolumn{7}{|l|}{ Hospital volume, patients per year } \\
\hline $\operatorname{Low}(\leq 66)$ & $28,467(36)$ & $38,650(31)$ & 11 & $22,141(36)$ & $22,355(36)$ & 1 \\
\hline Medium (67-129) & $28,609(36)$ & $39,238(32)$ & 10 & $21,325(34)$ & $21,692(35)$ & 1 \\
\hline $\operatorname{High}(\geq 130)$ & $21,570(27)$ & $46,332(37)$ & 21 & $18,886(30)$ & 18,305 (29) & 2 \\
\hline
\end{tabular}

Data are presented as $\mathrm{n}(\%)$ or mean \pm standard deviation 


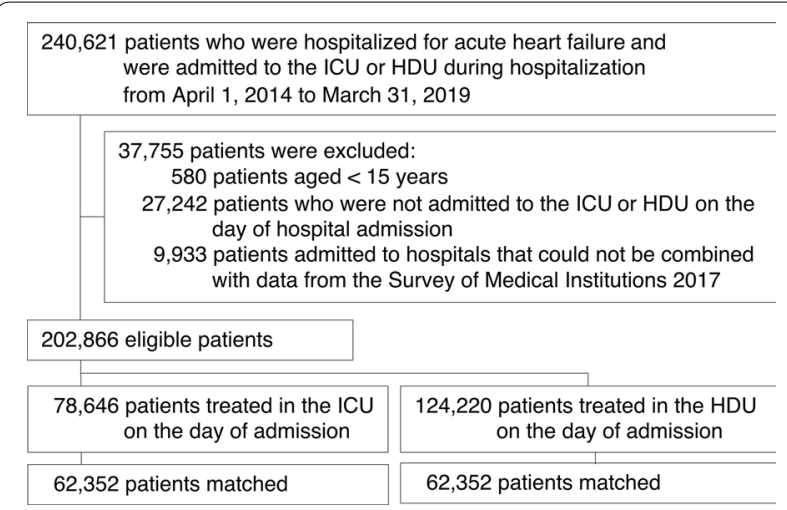

Fig. 1 Patient flowchart. ICU, intensive care unit; HDU, high-dependency care unit

patients in the ICU group tended to be younger; be in a comatose state; use an ambulance; require noninvasive ventilation, intubation, nitrates, nicorandil, dobutamine, noradrenaline, intra-aortic balloon pumping, coronary angiography, percutaneous coronary intervention, and continuous renal replacement therapy; and be admitted to an academic hospital. In contrast, patients in the HDU group tended to have severe dependence, have dementia, be admitted from a nursing home, have atrial flutter/ fibrillation, be admitted to a tertiary emergency hospital, and be admitted to a high-volume hospital. One-to-one propensity score matching created 62,352 matched pairs. The distributions of propensity scores before and after the matching are shown in Additional file 1: Figs. S1 and S2. After the propensity score matching, the patients' characteristics were well balanced between the two groups (Table 1 and Additional file 1: Fig. S3).
Table 2 shows the outcomes before and after the propensity score matching. After the propensity score matching, there was no statistically significant difference in in-hospital mortality between the ICU and HDU groups $(10.7 \%$ vs. $11.4 \%$; difference, $-0.6 \%$; $95 \%$ confidence interval, $-1.5 \%$ to $0.2 \%$ ). Compared with patients in the HDU group, those in the ICU group had significantly longer lengths of hospital stay and higher hospitalization costs. There were no statistically significant differences between the two groups in the length of ICU/ HCU stay or complications after admission except stroke.

The results of the subgroup analyses in the propensity score-matched cohort are shown in Table 3. There was a statistically significant difference in in-hospital mortality between the ICU and HDU groups among patients receiving noninvasive ventilation $(9.4 \%$ vs. $10.5 \%$; difference, $-1.0 \%$; $95 \%$ confidence interval, $-1.9 \%$ to $-0.1 \%$ ) and patients receiving intubation $(32.5 \%$ vs. $40.6 \%$; difference, $-8.0 \%$; $95 \%$ confidence interval, $-14.5 \%$ to $-1.5 \%$ ). There were no statistically significant differences in inhospital mortality between the two groups in other subgroup analyses.

Of the 202,866 patients in the 737 hospitals with ICU and/or HDU beds, 19,512 (10\%) were admitted to 157 hospitals with only ICU beds, 23,310 (11\%) were admitted to 186 hospitals with only HDU beds, and 160,044 (79\%) were admitted to 394 hospitals with both ICU and HDU beds. The results of the sensitivity analyses excluding patients admitted to hospitals with both ICU and HDU beds are shown in Table 4. There were no statistically significant differences between the two groups in in-hospital mortality, length of hospital stay, and complications after admission. Compared with patients in

Table 2 Outcomes before and after propensity score matching

\begin{tabular}{|c|c|c|c|c|c|c|}
\hline \multirow[t]{2}{*}{ Outcomes } & \multicolumn{2}{|c|}{$\begin{array}{l}\text { Before propensity score } \\
\text { matching }\end{array}$} & \multicolumn{2}{|c|}{ After propensity score matching } & \multirow[t]{2}{*}{$\begin{array}{l}\text { Difference } \\
(95 \% \mathrm{Cl})\end{array}$} & \multirow[t]{2}{*}{$P$ value } \\
\hline & $\begin{array}{l}\text { ICU } \\
(n=78,646)\end{array}$ & $\begin{array}{l}\text { HDU } \\
(n=124,220)\end{array}$ & $\begin{array}{l}\text { ICU } \\
(n=62,352)\end{array}$ & $\begin{array}{l}\text { HDU } \\
(n=62,352)\end{array}$ & & \\
\hline In-hospital mortality & $8,759(11.1)$ & $14,672(11.8)$ & $6,696(10.7)$ & $7,101(11.4)$ & $-0.6(-1.5$ to 0.2$)$ & 0.14 \\
\hline Length of hospital stay, days & $26 \pm 31$ & $24 \pm 24$ & $25 \pm 28$ & $24 \pm 25$ & $1.1(0.3$ to 1.9$)$ & 0.006 \\
\hline Length of ICU/HDU stay, days & $5 \pm 9$ & $5 \pm 6$ & $5 \pm 9$ & $5 \pm 7$ & -0.1 ( -0.4 to 0.2$)$ & 0.42 \\
\hline Total hospitalization cost, USD & $17,670(29,246)$ & $12,924(15,891)$ & $15,934(23,606)$ & $13,751(18,347)$ & 2183 (1435 to 2931) & $<0.001$ \\
\hline \multicolumn{7}{|l|}{ Complications after admission } \\
\hline Pneumonia & $3,199(4.1)$ & $4,040(3.3)$ & $2,356(3.8)$ & $2,155(3.5)$ & $0.3(-0.1$ to 0.7$)$ & 0.13 \\
\hline Stroke & $2,124(2.7)$ & $2,709(2.2)$ & $1,631(2.6)$ & $1,382(2.2)$ & $0.4(0.1$ to 0.7$)$ & 0.003 \\
\hline Endoscopic hemostasis for Gl bleeding & $417(0.5)$ & $563(0.5)$ & $320(0.5)$ & $313(0.5)$ & $0.01(-0.07$ to 0.09$)$ & 0.79 \\
\hline Catheter-related bloodstream infection & $111(0.1)$ & $92(0.1)$ & $79(0.1)$ & $56(0.1)$ & 0.04 (0.00 to 0.07$)$ & 0.050 \\
\hline Clostridioides difficile infection & $223(0.3)$ & $319(0.3)$ & $183(0.3)$ & $153(0.2)$ & $0.05(-0.04$ to 0.01$)$ & 0.28 \\
\hline
\end{tabular}

Data are presented as $\mathrm{n}(\%)$ or mean \pm standard deviation

ICU intensive care unit; HDU high-dependency care unit; $C l$ confidence interval; USD United States dollars; GI gastrointestinal 
Table 3 Results of subgroup analyses for in-hospital mortality

\begin{tabular}{|c|c|c|c|c|}
\hline \multirow[t]{2}{*}{ Analyses } & \multicolumn{2}{|c|}{ In-hospital mortality, n (\%) } & \multirow{2}{*}{$\begin{array}{l}\text { Difference, \% } \\
(95 \% \mathrm{Cl})\end{array}$} & \multirow[t]{2}{*}{$P$ value } \\
\hline & ICU & HDU & & \\
\hline Overall cohort & $6,696 / 62,352(10.7)$ & $7,101 / 62,352(11.4)$ & $-0.6(-1.5$ to 0.2$)$ & 0.14 \\
\hline \multicolumn{5}{|l|}{ Subgroups } \\
\hline \multicolumn{5}{|l|}{ Respiratory support } \\
\hline No supplemental oxygen & $1,087 / 12,005(9.1)$ & $1,055 / 12,106(8.7)$ & $0.3(-0.8$ to 1.4$)$ & 0.54 \\
\hline Supplemental oxygen & 2,393/24,121 (9.9) & $2,241 / 23,535(9.5)$ & $0.4(-0.3$ to 1.1$)$ & 0.29 \\
\hline Noninvasive ventilation & $2,171 / 23,015(9.4)$ & $2,442 / 23,351(10.5)$ & $-1.0(-1.9$ to -0.1$)$ & 0.026 \\
\hline Intubation & $1,045 / 3,211(32.5)$ & $1,363 / 3,360(40.6)$ & $-8.0(-14.5$ to -1.5$)$ & 0.015 \\
\hline \multicolumn{5}{|l|}{ Intravenous vasodilator } \\
\hline Yes & $2,958 / 42,058(7.0)$ & $3,146 / 41,971(7.5)$ & $-0.5(-1.0$ to 0.1$)$ & 0.097 \\
\hline No & 3,738/20,294 (18.4) & 3,955/20,381 (19.4) & $-1.0(-3.1$ to 1.1$)$ & 0.36 \\
\hline \multicolumn{5}{|l|}{ Diuretic } \\
\hline Yes & $3,873 / 43,123(9.0)$ & $4,010 / 43,155(9.3)$ & -0.3 ( -0.9 to 0.3$)$ & 0.30 \\
\hline No & 2,823/19,229 (14.7) & $3,091 / 19,197(16.1)$ & $-1.4(-3.6$ to 0.8$)$ & 0.21 \\
\hline \multicolumn{5}{|l|}{ Inotrope } \\
\hline Yes & $1,527 / 8,735(17.5)$ & $1,549 / 8,843(17.5)$ & $0.0(-1.5$ to 1.4$)$ & 0.96 \\
\hline No & $5,169 / 53,617(9.6)$ & $5,552 / 53,509(10.4)$ & $-0.7(-1.7$ to 0.2$)$ & 0.13 \\
\hline \multicolumn{5}{|l|}{ Vasopressor } \\
\hline Yes & $1,434 / 5,047(28.4)$ & 1,522/4,985 (30.5) & $-2.1(-4.4$ to 0.2$)$ & 0.070 \\
\hline No & $5,262 / 57,305(9.2)$ & $5,579 / 57,367(9.7)$ & $-0.5(-1.4$ to 0.3$)$ & 0.19 \\
\hline \multicolumn{5}{|c|}{ Mechanical circulatory support } \\
\hline Yes & $150 / 583(25.7)$ & $172 / 609(28.2)$ & $-2.5(-7.9$ to 2.9$)$ & 0.36 \\
\hline No & $6,546 / 61,769(10.6)$ & $6,929 / 61,743(11.2)$ & $-0.6(-1.5$ to 0.2$)$ & 0.16 \\
\hline \multicolumn{5}{|l|}{ Renal replacement therapy } \\
\hline Yes & $306 / 3,052(10.0)$ & 282/3,087 (9.1) & $0.9(-0.8$ to 2.6$)$ & 0.31 \\
\hline No & $6,390 / 59,300(10.8)$ & $6,819 / 59,265(11.5)$ & $-0.7(-1.6$ to 0.1$)$ & 0.12 \\
\hline
\end{tabular}

ICU intensive care unit; $H D U$ high-dependency care unit; $C I$ confidence interval

Table 4 Results of sensitivity analyses excluding patients admitted to hospitals with both ICU and HDU beds after propensity score matching

\begin{tabular}{|c|c|c|c|c|}
\hline \multirow[t]{3}{*}{ Outcomes } & \multicolumn{4}{|c|}{ After propensity score matching } \\
\hline & ICU & HDU & Difference & $P$ value \\
\hline & $(n=11,527)$ & $(n=11,527)$ & $(95 \% \mathrm{Cl})$ & \\
\hline In-hospital mortality & $1,465(12.7)$ & $1,480(12.8)$ & $-0.1(-1.9$ to 1.6$)$ & 0.88 \\
\hline Length of hospital stay, days & $27 \pm 26$ & $28 \pm 27$ & $-0.9(-2.9$ to 1.0$)$ & 0.33 \\
\hline Length of ICU/HDU stay, days & $5 \pm 7$ & $5 \pm 7$ & $-0.9(-1.4$ to -0.5$)$ & $<0.001$ \\
\hline Total hospitalization cost, USD & $14,501(17,070)$ & $12,584(11,462)$ & 1916 (918 to 2914) & $<0.001$ \\
\hline \multicolumn{5}{|l|}{ Complications after admission } \\
\hline Pneumonia & $431(3.7)$ & $496(4.3)$ & $-0.6(-1.4$ to 0.3$)$ & 0.18 \\
\hline Stroke & $293(2.5)$ & $253(2.2)$ & $0.3(-0.2$ to 0.9$)$ & 0.24 \\
\hline Endoscopic hemostasis for Gl bleeding & $53(0.5)$ & $59(0.5)$ & $-0.05(-0.24$ to 0.13$)$ & 0.58 \\
\hline Catheter-related bloodstream infection & $21(0.2)$ & $13(0.1)$ & $0.07(-0.03$ to 0.17$)$ & 0.18 \\
\hline Clostridioides difficile infection & $46(0.4)$ & $47(0.4)$ & $0.00(-0.25$ to 0.23$)$ & 0.94 \\
\hline
\end{tabular}

Data are presented as $\mathrm{n}(\%)$ or mean \pm standard deviation

ICU intensive care unit; HDU high-dependency care unit; $C l$ confidence interval; USD United States dollars; GI gastrointestinal 
the HDU group, those in the ICU group had significantly shorter lengths of ICU/HCU stay and higher hospitalization costs.

Of 78,646 patients who were admitted to the ICU on the day of admission, 9,747 (12\%) were admitted to resource-rich ICUs and 68,899 (88\%) were admitted to standard ICUs. The results of the sensitivity analyses between patients in resource-rich ICUs versus HDUs, standard ICUs versus HDUs, and resource-rich ICUs versus standard ICUs were also similar to those of the main analyses (Table 5).

\section{Discussion}

In this nationwide cohort study of patients with acute heart failure, there was no significant difference in inhospital mortality between the ICU and HDU groups in the entire cohort. This finding was consistent in the sensitivity analyses comparing structure and staffing models with different intensivist staffing models and nurse-topatient ratios. Meanwhile, the ICU group had significantly lower in-hospital mortality than the HDU group among patients receiving noninvasive ventilation and intubation.

Unlike previous studies on general ICUs [25], one previous study on CICUs [13], and recommendations from academic societies $[4,8-10]$, the present study showed that the different structure and staffing models were not associated with reduced mortality in patients with acute heart failure. One possible reason is that intensivist staffing failed to produce a benefit over specialized care by cardiologists for patients with acute heart failure. Intensivists have the potential to improve patient care and outcomes through their specialist knowledge of organ support therapies, extensive experience with critically ill patients, and higher compliance with evidence-based protocols [26]. However, most of the patients in the present cohort did not require organ support therapies including invasive mechanical ventilation, mechanical circulatory support, and renal replacement therapy. Therefore, the specialty of intensivists might not be utilized.

Another possible reason is that a nurse-to-patient ratio of 1:4 or 1:5 was not inferior to a ratio of $1: 2$ in the present cohort of patients with acute heart failure. Previous studies have shown that inadequate nurse staffing is associated with increased mortality and that critically ill patients demand high nurse workloads $[27,28]$. However, because most of the patients in the present cohort only required single organ support for respiratory failure or circulatory failure without invasive treatments, a nurse-to-patient ratio of 1:4 may be adequate for care of such patients requiring a lower workload.

The above reasons may also explain the lower mortality in the ICU group in the subgroup of patients receiving noninvasive ventilation and intubation. The recent ICU admission guideline recommends that patients with invasive treatments such as mechanical ventilation have the highest priority for ICU admission [7]. Therefore, this study may support ICU triage based on the combination of patient type and invasive interventions rather than based on the diagnosis alone.

The findings of this study should be interpreted carefully. Because this was not a clinical trial, no causation can be inferred. The findings of this study do not support the treatment of all patients with acute heart failure in the HDU instead of the ICU. In critically ill patients, overtriage is recommended and preferable to undertriage

Table 5 Results of sensitivity analyses of in-hospital mortality between patients in resource-rich ICUs versus HDUs, standard ICUs versus HDUs, and resource-rich ICUs versus standard ICUs

\begin{tabular}{|c|c|c|c|}
\hline \multirow[t]{3}{*}{ Sensitivity analyses } & \multicolumn{3}{|c|}{ After propensity score matching } \\
\hline & In-hospital & Difference, \% & $P$ value \\
\hline & Mortality, $n(\%)$ & $(95 \% \mathrm{Cl})$ & \\
\hline \multicolumn{4}{|c|}{ Resource-rich ICU vs. HDU } \\
\hline Resource-rich ICU & 956/9661 (9.9) & $-1.1(-2.4$ to 0.4$)$ & 0.16 \\
\hline HDU & 1065/9661 (11.0) & & \\
\hline \multicolumn{4}{|l|}{ Standard ICU vs. HDU } \\
\hline Standard ICU & $6272 / 57,384(10.9)$ & $-0.6(-1.5$ to 0.3$)$ & 0.22 \\
\hline $\mathrm{HDU}$ & $6596 / 57,384(11.5)$ & & \\
\hline \multicolumn{4}{|c|}{ Resource-rich ICU vs. standard ICU } \\
\hline Resource-rich ICU & $986 / 9745(10.1)$ & $-1.2(-2.6$ to 0.2$)$ & 0.085 \\
\hline Standard ICU & $1106 / 9745(11.3)$ & & \\
\hline
\end{tabular}

ICU intensive care unit; $H D U$ high-dependency care unit; $\mathrm{Cl}$ confidence interval 
[7]. Therefore, the present study shows one possibility that care in the HDU for patients with non-advanced acute heart failure may be cost-effective without compromising quality. Further studies are needed to verify our findings and to examine how other structure and staffing models of CICUs impact patient outcomes.

The present study has some limitations. First, we used a multicenter, real-world database in Japan, and there was no standard protocol for critical care admission. Therefore, admission to the HDU rather than the ICU for patients with acute heart failure was not random and was based on the decision of the attending physicians or circumstances of each hospital, which may have led to confounding by indication. We attempted to control for measured confounders in the propensity score analyses; however, there still may have been unmeasured confounders such as vital signs [29], prior hospitalization for heart failure [30] and ejection fraction [31, 32]. Therefore, we conducted a sensitivity analysis excluding patients admitted to hospitals with both ICU and HDU beds and confirmed that the impact of this bias would be small. Second, because the severity of illness and invasive interventions might modify the effect of ICU admission on in-hospital mortality, the average effect will differ between different populations. Furthermore, the definitions of ICU and HDU are not consistent among countries. Therefore, the results of this study may not be generalizable to other populations of patients who receive care in the CICU. Third, there are other unmeasured factors that affect assessment of organizational structure and staffing models, such as closed or open ICU models [5], the presence of cardiac intensivists, general ICUs or cardiac-specialized ICUs, the number of full-time or nonfull-time doctors, and physician's specialty (cardiologist or intensivist). Therefore, future studies should include these variables to clarify which organizational structure and staffing models are most effective in reducing mortality.

\section{Conclusion}

The present study showed that care in the ICU was not associated with lower in-hospital mortality than care in the HDU among the entire cohort with acute heart failure. However, critical care in ICUs was associated with lower in-hospital mortality than critical care in HDUs among patients receiving noninvasive ventilation and intubation.

\section{Abbreviations}

CICU: Cardiac intensive care unit; ICU: Intensive care unit; HDU: High-dependency care unit; ICD-10: International Classification of Diseases, Tenth Revision.

\section{Supplementary Information}

The online version contains supplementary material available at https://doi. org/10.1186/s40560-021-00592-2.

Additional file 1: Table S1. Japanese medical procedure codes used to define ICUs and HDUs. Fig. S1. Distributions of propensity scores before propensity score matching in the main analysis. Fig. S2. Distributions of propensity scores after propensity score matching in the main analysis. Fig. S3. Balance of the covariates before and after propensity score matching in the main analysis

\section{Acknowledgements}

Not applicable.

\section{Author contributions}

$\mathrm{HO}$ designed the research; $\mathrm{HO}$ and $\mathrm{HM}$ conducted the research; $\mathrm{HO}$ and $\mathrm{HY}$ analyzed the data; $\mathrm{HO}, \mathrm{HM}$, and $\mathrm{HY}$ wrote the paper; and $\mathrm{HO}$ had primary responsibility for the final content. All authors read and approved the final manuscript.

\section{Funding}

This work was supported by grants from the Ministry of Health, Labour and Welfare, Japan (Grant Nos. 21 AA2007 and 20AA2005) and the Ministry of Education, Culture, Sports, Science and Technology, Japan (Grant No. 20H03907).

\section{Availability of data and materials}

The dataset analyzed in the current study is not publicly available because of contracts with the hospitals providing data to the database.

\section{Declarations}

\section{Ethics approval and consent to participate}

The Institutional Review Board of The University of Tokyo approved this study. The procedures used in this study adhere to the tenets of the Declaration of Helsinki. No information allowing the identification of individual patients, hospitals, or physicians was obtained, and the requirement for informed consent was waived because of the anonymous nature of the data.

\section{Consent for publication}

Not applicable.

\section{Competing interests}

The authors declare that they have no competing interests.

Received: 30 October 2021 Accepted: 10 December 2021

Published online: 20 December 2021

\section{References}

1. Katz JN, Shah BR, Volz EM, et al. Evolution of the coronary care unit: clinical characteristics and temporal trends in healthcare delivery and outcomes. Crit Care Med. 2010;38:375-81.

2. Bohula EA, Katz JN, van Diepen S, et al. Demographics, care patterns, and outcomes of patients admitted to cardiac intensive care units: the Critical Care Cardiology Trials Network Prospective North American Multicenter Registry of Cardiac Critical IIIness. JAMA Cardiol. 2019;4:928-35.

3. Casella G, Cassin M, Chiarella F, et al. BLITZ-3 Investigators: epidemiology and patterns of care of patients admitted to Italian intensive cardiac care units: the BLITZ-3 registry. J Cardiovasc Med (Hagerstown). 2010:11:450-61.

4. Morrow DA, Fang JC, Fintel DJ, et al. American Heart Association Council on Cardiopulmonary, Critical Care, Perioperative and Resuscitation, Council on Clinical Cardiology, Council on Cardiovascular Nursing, and Council on Quality of Care and Outcomes Research: Evolution of critical care cardiology: transformation of the cardiovascular intensive care unit 
and the emerging need for new medical staffing and training models: a scientific statement from the American Heart Association. Circulation. 2012;126:1408-28.

5. Pronovost PJ, Angus DC, Dorman T, et al. Physician staffing patterns and clinical outcomes in critically ill patients: a systematic review. JAMA. 2002;288:2151-62.

6. Valentin A, Ferdinande P. ESICM Working Group on Quality Improvement: recommendations on basic requirements for intensive care units: structural and organizational aspects. Intensive Care Med. 2011:37:1575-87.

7. Nates JL, Nunnally M, Kleinpell R, et al. ICU admission, discharge, and triage guidelines: a framework to enhance clinical operations, development of institutional policies, and further research. Crit Care Med. 2016:44:1553-602.

8. Tsutsui $\mathrm{H}$, Isobe $\mathrm{M}$, Ito $\mathrm{H}$, et al. Japanese Circulation Society and the Japanese Heart Failure Society Joint Working Group: JCS 2017/JHFS 2017 Guideline on diagnosis and treatment of acute and chronic heart failure digest version. Circ J. 2019;83:2084-184.

9. JSICM Investigative Committee for standards for establishing intensive care units. CCU establishment guidelines. J Jpn Soc Intensive Care Med. 2004:11:259-267.

10. Hasin Y, Danchin N, Filippatos GS, et al. Working Group on Acute Cardiac Care of the European Society of Cardiology: Recommendations for the structure, organization, and operation of intensive cardiac care units. Eur Heart J. 2005;26:1676-82.

11. van Diepen $S$, Granger $C B$, Jacka $M$, et al. The unmet need for addressing cardiac issues in intensive care research. Crit Care Med. 2015;43:128-34.

12. Katz JN, Minder M, Olenchock B, et al. The genesis, maturation, and future of critical care cardiology. J Am Coll Cardiol. 2016;68:67-79.

13. $\mathrm{Na} \mathrm{SJ}$, Chung CR, Jeon $\mathrm{K}$, et al. Association between presence of a cardiac intensivist and mortality in an adult cardiac care unit. J Am Coll Cardiol. 2016:68:2637-48.

14. Shmueli A, Sprung CL. Assessing the in-hospital survival benefits of intensive care. Int J Technol Assess Health Care. 2005;21:66-72.

15. Ohbe $H$, Sasabuchi $Y$, Kumazawa R, et al. Intensive care unit occupancy in Japan, 2015-2018: a nationwide inpatient database study. J Epidemiol. 2021. https://doi.org/10.2188/jea.JE20210016.

16. Yasunaga $\mathrm{H}$. Real world data in Japan: Chapter II. The diagnosis procedure combination database. Ann Clin Epidemiol. 2019:1:76-9.

17. Yamana H, Moriwaki M, Horiguchi $\mathrm{H}$, et al. Validity of diagnoses, procedures, and laboratory data in Japanese administrative data. J Epidemiol. 2017;27:476-82

18. The Ministry of Health, Labour and Welfare, Japan statistical surveys 2017. 2017. https://www.mhlw.go.jp/stf/seisakunitsuite/bunya/open_data. html. Accessed June 2, 2021.

19. Prin M, Wunsch H. The role of stepdown beds in hospital care. Am J Respir Crit Care Med. 2014;190:1210-6.

20. Boots R, Lipman J. High dependency units: issues to consider in their planning. Anaesth Intensive Care. 2002;30:348-54.

21. Shigematsu K, Nakano H, Watanabe $Y$. The eye response test alone is sufficient to predict stroke outcome-reintroduction of Japan Coma Scale: a cohort study. BMJ Open. 2013;3:e002736.

22. Mahoney FI, Barthel DW. Functional evaluation: the Barthel Index. Md State Med J. 1965:14:61-5.

23. Rosenbaum PR, Rubin DB. Constructing a control group using multivariate matched sampling methods that incorporate the propensity score. Am Stat. 1985;39:33-8.

24. Austin PC. Balance diagnostics for comparing the distribution of baseline covariates between treatment groups in propensity-score matched samples. Stat Med. 2009;28:3083-107.

25. Wilcox ME, Chong CAKY, Niven DJ, et al. Do intensivist staffing patterns influence hospital mortality following ICU admission? A systematic review and meta-analyses. Crit Care Med. 2013:41:2253-74.

26. Kahn JM, Brake H, Steinberg KP. Intensivist physician staffing and the process of care in academic medical centres. Qual Saf Health Care. 2007;16:329-33.

27. Needleman J, Buerhaus P, Pankratz VS, et al. Nurse staffing and inpatient hospital mortality. N Engl J Med. 2011;364:1037-45.

28. Penoyer DA. Nurse staffing and patient outcomes in critical care: a concise review. Crit Care Med. 2010;38:1521-8.
29. Mebazaa A, Gheorghiade M, Piña IL, et al. Practical recommendations for prehospital and early in-hospital management of patients presenting with acute heart failure syndromes. Crit Care Med. 2008:36:S129-39.

30. Setoguchi S, Stevenson LW, Schneeweiss S. Repeated hospitalizations predict mortality in the community population with heart failure. Am Heart J. 2007;154:260-6.

31. Yancy CW, Jessup M, Bozkurt B, et al. American College of Cardiology Foundation/American Heart Association Task Force on Practice Guidelines: 2013 ACCF/AHA guideline for the management of heart failure: a report of the American College of Cardiology Foundation/ American Heart Association Task Force on practice guidelines. Circulation. 2013;128:e240-327.

32. Ponikowski P, Voors AA, Anker SD, et al. ESC Guidelines for the diagnosis and treatment of acute and chronic heart failure: The Task Force for the diagnosis and treatment of acute and chronic heart failure of the European Society of Cardiology (ESC). Developed with the special contribution of the Heart Failure Association (HFA) of the ESC. Eur J Heart Fail. 2016;18:891-975.

\section{Publisher's Note}

Springer Nature remains neutral with regard to jurisdictional claims in published maps and institutional affiliations.

Ready to submit your research? Choose BMC and benefit from:

- fast, convenient online submission

- thorough peer review by experienced researchers in your field

- rapid publication on acceptance

- support for research data, including large and complex data types

- gold Open Access which fosters wider collaboration and increased citations

- maximum visibility for your research: over $100 \mathrm{M}$ website views per year

At BMC, research is always in progress.

Learn more biomedcentral.com/submissions 